\title{
Viable ovarian pregnancy: case report
}

\begin{abstract}
Ovarian pregnancy is a rare variable of ectopic pregnancy with an incidence is $1-3 \%$ of all ectopic pregnancies. It still remains a diagnostic challenge. As the ovarian pregnancy clinical presentation is similar to that of tubal one, and an accurate ultrasound diagnosis is someway controversial, the surgical diagnosis is frequently made and confirmed by histo-pathological examination. We are presenting the data of two cases of viable ovarian pregnancies presented with hemodynamic instability that required immediate laparotomies. Both cases needed unilateral salpingo-oophrectomy. These clinical scenarios stresses on the necessity of starting early antenatal care and having a routine transvaginal first trimester ultrasound. Also clear evidence based guideline for ovarian pregnancy management should be initiated using the best available data on the literature.
\end{abstract}

Keywords: ectopic pregnancy; ovarian pregnancy; laparotomy
Volume 4 Issue I - 2017

\author{
Ahmed Altraigey, ${ }^{1,2}$ Wael Naeem,' Omar \\ Khaled,' Mufareh Asiri, ${ }^{2}$ Abdullah Asiri, ${ }^{3}$ \\ Mohammed Hussein ${ }^{2}$ \\ 'Department of Obstetrics and Gynecology, Benha University, \\ Egypt \\ ${ }^{2}$ Department of Obstetrics and Gynecology,Armed Forces \\ Hospitals Southern Region, Saudi Arabia \\ ${ }^{3}$ Department of Obstetrics and Gynecology, King Khalid \\ University, Saudi Arabia
} and Gynecology, King Faisal Military City, base villa 9, Khamis Mushayt, 61961, Kingdom of Saudi Arabia - 43 Benha-Zagazig Street, Mansheyet Elnoor, Benha, I35 I I,Arab Republic of Egypt, Egypt, Tel +966544854232, +20I060885050,

Email ahmed.altraigey@yahoo.com; ahmed.abdelfattah@fmed. bu.edu.eg

Received: December 18, 2016 | Published: January 03, 2017

\section{Introduction}

The incidence of ectopic pregnancy is on the rise due to increasing use of ovulation induction and assisted reproduction techniques. ${ }^{1,2}$ Ovarian pregnancy is uncommon form of ectopic pregnancy with an incidence of 1/7000-1/40,000 live births. ${ }^{3}$ Approximately $75 \%$ terminate in first trimester and are often misdiagnosed as corpus luteum hemorrhage. ${ }^{4}$ Role of ultrasound in diagnosis of ovarian gestation had been described but most of the women present with ruptured ectopic and are in circulatory collapse so preoperative diagnosis of ovarian ectopic on sonography is not easy. ${ }^{5,6}$ The delay in diagnosis of ovarian pregnancies has been attributed to the non-specific clinical presentation upon admission, while the massive intra-abdominal bleeding has been explained by the fragility and hypervascularity of the ovarian tissue..$^{7-9}$

\section{Case reports}

\section{Case I}

A 28year old woman G4P2+1 with history of previous two lower segment caesarean sections (LSCS) and the last was one year ago. She presented to emergency room of Benha University hospitals with missed period of 8 weeks and lower abdominal pain mainly on right side. Woman was using combined oral contraceptive pills for one year. On general examination, her blood pressure was 90/60mmhg, pulse $122 \mathrm{bpm}$ and temperature of $37.2^{\circ} \mathrm{C}$. On abdominal examination, she showed tenderness and rebound tenderness on right iliac fossa. Ultrasound was done showing empty uterus, mild free fluid cc"11ection in Douglas Pouch with right adenxal cystic mass about $\left(6^{\times} 4 \mathrm{~cm}\right)$ with single fetal pole and pulsating heart. Her blood work revealed hemoglobin $(\mathrm{Hb})$ of $10.5 \mathrm{gm} / \mathrm{dl}$. Couple was counseled about the management plan. Packed red blood cells (RBCs) were crossmatched; complete informed consent was signed before proceeding to emergency laparotomy. Intra-operatively, left adenxa was free with mild intra-peritoneal collection, adhesion of intestine to back of uterus and left tube making it difficult to explore the whole pelvis. The general surgeon was called and meticulous dissection of adhesion was performed. This freed the pelvis to show presence of right ovarian ectopic pregnancy (Figure 1). Right salpingo-oophorectomy was done with insertion of intra peritoneal drain. Postoperatively, her vital signs urinary output and laboratory results were satisfactory. And the drain was removed after total output of $100 \mathrm{ml}$ bloody fluid. Woman was discharged on day 3 postoperative in good condition and her serum human chorionic gonadotrophin $(\beta \mathrm{hCG})$ level was followed in outpatient clinic till became negative on $3^{\text {rd }}$ week postoperative. She was offered contraception in the form of medroxy-progesterone acetate injection. Histopathology showed ovarian tissue in the gestational sac.

\section{Case 2}

A 35year old woman, G4P2+1 was admitted to our hospital with complain of lower abdominal pain mainly on right side. Woman was known to be pregnant at 6weeks of gestation according to her dates. Patient is known to be hypertensive and asthmatic and history of one LSCS. Serum pregnancy test was positive. Her vital signs were recorded and revealed blood pressure of 100/50mmhg, pulse of $125 \mathrm{bpm}$ and temperature of $36.8^{\circ} \mathrm{C}$. Abdominal examination showed tenderness and rebound tenderness on right iliac fossa. Ultrasound was done showing; empty uterus, mild free fluid collection in Douglas 
Pouch, right adenxal cystic mass about $\left(7^{\times} 6 \mathrm{~cm}\right)$ and single fetal pole $(\mathrm{CRL}=11 \mathrm{wk})$ with pulsated heart within. Her laboratory results showed $\mathrm{Hb}$ of $12.5 \mathrm{gm} / \mathrm{dl}$ and normal renal and liver function tests. Counseling for the couple was done as well as cross-matching packed RBCs. Informed consent was obtained before proceeding to laparotomy. Intraoperatively, intraperitoneal collection was taken out through suction and disturbed gestational sac with fetus inside within Douglas Pouch were found to be attached to right tubal fimbria, back of the uterus and the left ovary (Figure 2). Right partial salpingectomy was performed with preservation of the right ovary. An excision of some trophoplastic omental patches was done as well as the fetus and both were sent for histopathological examination (Figure 3). Finally, insertion of large intraperitoneal drain was done. Postoperatively, her vital signs urinary output and laboratory results were satisfactory. And the drain was removed after total output of $200 \mathrm{ml}$ bloody fluid. Woman was discharged on day 4 postoperative in good condition and her serum human chorionic gonadotrophin ( $\beta \mathrm{hCG}$ ) level was followed in outpatient clinic till became negative on $4^{\text {th }}$ week postoperative. She was offered contraception in the form of medroxy-progesterone acetate injection. Histopathology showed ovarian tissue in the gestational sac.

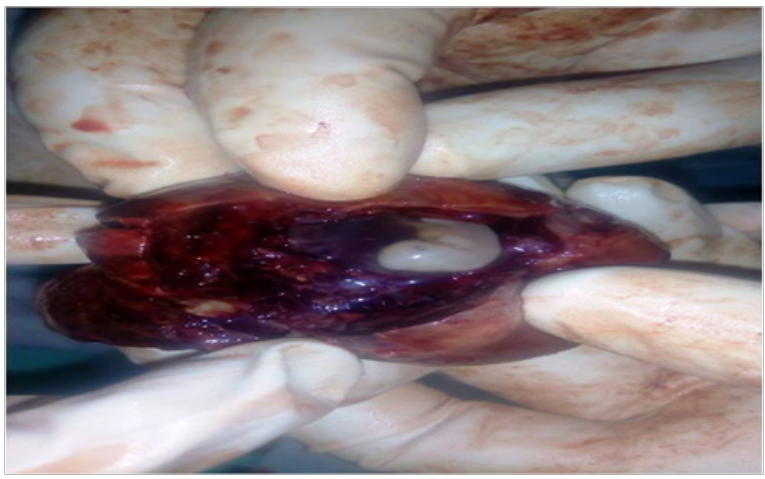

Figure I Showing the embryo inside the gestational sac within the excised ovary.

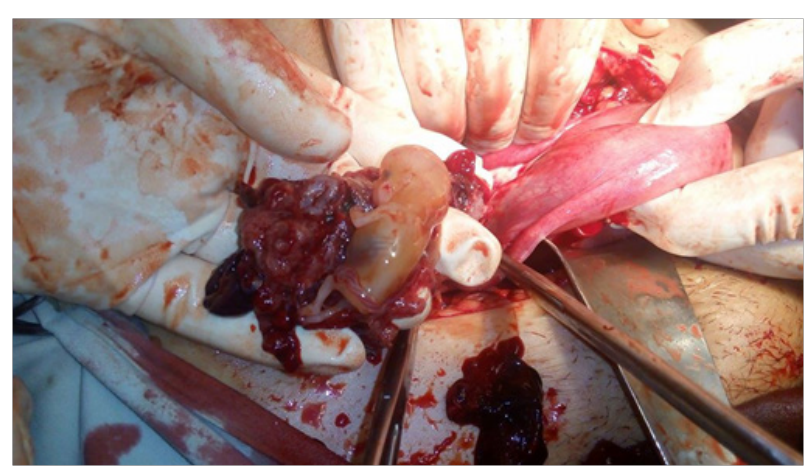

Figure 2 Showing fetus attached to the gestational sac inside the excised ovary. The fallopian tube is intact in the background.

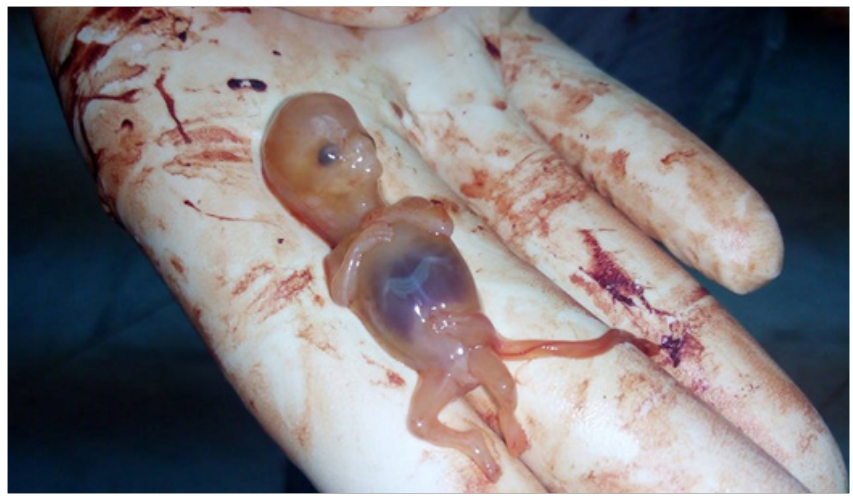

Figure 3 Showing fetus after being separated from the ovarian pregnancy.

\section{Discussion and literature review}

Primary ovarian pregnancy is a rare finding; it was first described by Dr. Saint Monnissey in $1682 .{ }^{10}$ Its reported incidence is 0.15 $3 \%$ of extrauterine gestations. ${ }^{11}$ It is either primary or secondary. Primary type occurs if the ovum is fertilized while still within the follicle while, secondary type occurs if fertilization took place in the ampullary part of the tube and the conceptus later implanted in the ovarian stroma. Furthermore, it can be classified to intrafollicular which is invariably primary or extrafollicular which may be primary or secondary where ovarian tissue is usually absent in the gestational sac. ${ }^{12}$ Although the explanation of implantation anomalies causing ovarian pregnancy remains unclear, there are various hypotheses such as: ovum liberation delay, tunica albuginea thickening, tubal dysfunction and intrauterine contraception devices (IUD). In contrast to women with tubal pregnancies, traditional risk factors; such as pelvic inflammatory disease and prior pelvic surgical procedure, may not play a role in ovarian pregnancy etiology. ${ }^{13}$ After a brief review of the literature, it seems that the primary ovarian pregnancy may occur without any classical risk factors and there is consensus that the only risk factor associated with the occurrence of ovarian pregnancy is the current use of IUD. ${ }^{9}$ Other risk factors reported seldom for ovarian pregnancy include endometriosis, sexually transmitted diseases, ovulation induction agents, tubal sterilization and a history of abdominal surgery. ${ }^{14}$

Both of the presented cases had history of previous major abdominal surgery in the form of LSCS. They presented with non-specific symptoms like lower abdominal pain and no vaginal bleeding. Their general examination after positive pregnancy test revealed hemodynamic instability with high shock index, so the initial diagnosis was disturbed ectopic pregnancy. Diagnosis is usually made using the Spiegelberg criteria (by Otto Spiegelberg ${ }^{15}$ ) which include: $:^{15}$ the gestational sac location is in the region of the ovary, the ectopic pregnancy is attached to the uterus by the ovarian ligament, histological proof that ovarian tissue is in the gestational sac wall and intact fallopian tube on the involved side. As these criteria are intra or postoperative findings, they should be combined with biochemical and ultrasonographic findings that include: serum human 
chorionic gonadotrophin $\beta$ hCG level $\geq 1000 \mathrm{IU} / \mathrm{L}$, empty uterine cavity at transvaginal ultrasound, normal tubes and absence of serum $\beta \mathrm{hCG}$ gradually after treatment of the ovarian pregnancy. ${ }^{16}$ Ultrasonographic evaluation of our cases showed empty uterus, adnexal mass and embryo with heart pulsation. There was no clear evidence that the tube is intact or that the ectopic pregnancy is ovarian. The suggestive findings of ovarian pregnancy on two-dimensional ultrasound which include; the absence of a yolk sac or fetal heart motion, a similar appearance to ruptured corpus luteum, explains the need for better and more accurate diagnostic markers. Comstock et al., ${ }^{6}$ evaluated the ultrasonographic appearance of diagnosed ectopic ovarian pregnancies. They showed a wide echogenic ring with an internal echolucent area as compared to a thin tubal ring with tubal pregnancies or corpus luteum cyst and occasionally a yolk sac or fetal heart motion were also identified. Three-dimensional ultrasound has been reported to distinguish ovarian pregnancy from corpus luteum cyst, which may improve detection. ${ }^{17}$ Although there were case reports explained conservative management and methotrexate therapy, our cases presented with hemodynamic instability which necessitates surgical intervension by laparotomy. The diagnosis is difficult and is a challenging to any gynecologist or surgeon and ovarian rupture destroys the integrity of the ovary and sometimes the fallopian tube making differentiation of such a gestation an obstacle. Thus, many case reports emphasize that in spite of the development of modern diagnostic methods, early and accurate diagnosis of primary ectopic ovarian pregnancy still needs to be improved. A proper protocol to identify ovarian pregnancy in early gestation will improve the treatment options that can be offered to affected women rather than the surgical option that is almost always required as the present with hemodynamic instability.

\section{Conclusion}

In conclusion, additional measurements are requested for early and accurate diagnosis of ovarian pregnancy which will allow several plans of management to be in place, thus changing the cascade of events. Recent ultrasound technologies may lead to earlier and precise detection of ovarian pregnancies allowing the use of non-invasive treatment modalities.

\section{Acknowledgements}

None.

\section{Conflict of interest}

The author declares no conflict of interest

\section{References}

1. Lewis G. The confidential enquiry into maternal and child health (CEMACH). Saving Mothers' Lives: reviewing maternal deaths to make motherhood safer-2003-2005. The seventh report on confidential enquiries into maternal deaths in the United Kingdom, UK: CEMACH; 2007. p. 1-296.
2. Dialani V, Levine D. Ectopic pregnancy: a review. Ultrasound $Q$. 2004;20(3):105-117.

3. Raziel A, Golan A, Pansky M, et al. Ovarian pregnancy: A report of twenty cases in one institution. Am J Obstet Gynecol. 1990;163(4 Pt 1):11821185 .

4. Hallatt JG. Primary ovarian pregnancy: a report of twenty-five cases. Am J Obstet Gynecol. 1982;143(1):55-60.

5. Dursun P, Gultekin M, Zeyneloglu HB. Ovarian ectopic pregnancy after ICSI-ET: a case report and literature review. Arch Gynecol Obstet. 2008;278(2):191-193.

6. Comstock C, Huston K, Lee W. The ultrasonographic appearance of ovarian ectopic pregnancies. Obstet Gynecol. 2005;105(1):42-45.

7. Chang FW, Chen $\mathrm{CH}$, Liu JY. Early diagnosis of ovarian pregnancy by ultrasound. Int J Gynecol Obstet. 2004;85(2):186-187.

8. Raziel A, Mordechai E, Schachter M, et al. A comparison of the incidence, presentation, and management of ovarian pregnancies between two periods of time. J Am Assoc Gynecol Laparosc. 2004;11(2):191-194.

9. Raziel A, Schachter M, Mordechai E, et al. Ovarian pregnancy-a 12-year experience of 19 cases in one institution. Eur J Obstet Gynecol Reprod Biol. 2004;114(1): 92-96.

10. Gerin-Lajoie L. Ovarian pegnancy. Am J Obstet Gynecol. 1951;62:920929.

11. Nwanodi $\mathrm{O}$, Khulpateea $\mathrm{N}$. The preoperative diagnosis of primary ovarian pregnancy. J Natl Med Assoc. 2006;98(5):796-798.

12. Gon S, Majumdar B, Ghosal T, et al. Two cases of primary ectopic ovarian pregnancy. Online J Health Allied Scs. 2011;10(1):26

13. Bagga R, Suri V, Verma P, et al. Failed medical management in ovarian pregnancy despite favorable prognostic factors - a case report. Med Gen Med. 2006;8(2):35.

14. Joseph RJ, Irvine LM. Ovarian ectopic pregnancy: Aetiology, diagnosis, and challenges in surgical management. $J$ Obstet Gynaecol. 2012;32(5):472-474.

15. Spiegelberg OX. Zur casuistic der ovarial schwangerschaft. Arch Gynekol. $1878 ; 13: 73$.

16. Sergent F, Mauger TF, Gravier A, et al. Ovarian pregnancies: revaluation of diagnostic criteria. Gynecol Obstet Biol Reprod (Paris). 2002;31(8):741746.

17. Ghi T, Banfi A, Marconi R, et al. Three-dimensional sonographic diagnosis of ovarian pregnancy. Ultrasound Obstet Gynecol. 2005;26(1):102-104. 